\title{
Fidel Castro tra storia, mito e demonologia
}

\author{
Duccio Basosi*
}

Fidel Castro: History, myth and demonology. The media all over the world met the news of the passing of Fidel Castro, on 25 November 2016, with extremely polarized commentaries on his legacy. This essay argues that historians in the future can contribute to a better understanding of those aspects and episodes of his long career as revolutionary and head of state that have made him such a controversial figure. At the same time, it appears that Fidel Castro fully belonged to the political left of the 20th century, as he constantly emphasized equality as the basis of all emancipation, and collective over individual subjects as far as instances of freedom were concerned. In that sense, polarized commentaries do not reflect misunderstandings about his life as much as diverging opinions on his politics.

Key words: Fidel Castro, Cuba, Cuban revolution, Communism, Third-worldism Parole chiave: Fidel Castro, Cuba, Rivoluzione cubana, Comunismo, Terzomondismo

A dispetto della diffusa idea che questa sia l'epoca del "mondo globalizzato", sono poche le notizie capaci di avere una risonanza davvero globale. Quella della morte di Fidel Castro, la sera del 25 novembre 2016, è stata tra queste: dal «Granma» al «New York Times», dal «Kommersant»al «Rénmín Rìbào», da «Le Monde» al «Mail \& Guardian», dalla BBC ad Al Jazeera, tutti gli organi informativi del mondo hanno non solo riferito del decesso dell'anziano comandante en jefe della rivoluzione cubana, ma anche affiancato la notizia con approfondimenti sulla sua vita, commenti e ampi servizi multimediali dalle strade di Cuba. A partire dai numerosi spunti emersi da tale lavoro giornalistico è possibile svolgere alcune brevi riflessioni sull'eredità politica del rivoluzionario e capo di stato cubano, così come questa si configura all'inizio del XXI secolo.

* Dipartimento di Studi linguistici e culturali comparati, Venezia; duccio.basosi@unive.it

«Passato e presente», a. XXXV (2017), n. 101, ISSN 1120-0650, ISSNe 1972-5493

DOI: $10.3280 / P A S S 2017-101002$ 
Non vi è dubbio, anzitutto, che "Fidel" (a Cuba nessuno lo ha mai chiamato "Castro") sia stato una figura di primo piano nella storia del '900, non solo di quella cubana e latinoamericana ma anche sulla scena globale. A tanta unanimità di giudizio sull'importanza storica del personaggio, tuttavia, non corrisponde un'analoga unanimità di giudizio sul suo operato: «liberatore» e «tiranno», «statista» e «avventuriero», «idealista» e «folle» sono solo alcuni degli aggettivi utilizzati dai commentatori nel tentativo di catturarne l'essenza ultima. Da questo punto di vista, i commenti post mortem hanno semplicemente riproposto la lunga storia di aspre controversie che hanno accompagnato Fidel lungo la maggior parte della sua vita, vale a dire dal momento in cui, con il lancio della riforma agraria nell'aprile 1959, il programma vagamente progressista della rivoluzione che lo aveva visto protagonista e líder cominciò ad assumere le forme di un'autentica azione di cambiamento sociale ed economico ${ }^{1}$. La virtuale unanimità di consensi (entusiastici a Cuba, curiosi in America Latina, rassegnati a Washington, sospettosi a Mosca e distratti in molte altre capitali mondiali) che ne aveva accompagnato l'ingresso trionfale all'Avana quattro mesi prima, iniziò allora a fratturarsi in modo irreparabile lungo le principali linee di faglia dei conflitti politici della seconda metà del ' 900 , per non trovare più una ricomposizione.

In questa sede, piuttosto che cercare di ricreare artificialmente l'unità perduta, appare quindi opportuno riconoscere che Fidel Castro è stato un "partigiano" e che riflettere sulla sua figura significa confrontarsi con tutti i principali conflitti che hanno costituito l'ossatura della storia politica mondiale della seconda metà del secolo scorso: dal conflitto Nord-Sud a quello EstOvest, dal conflitto di classe a quello di genere, a quello combattutosi lungo la "linea del colore".

Il punto di partenza di qualunque riflessione che ambisca a confrontarsi con questi temi non può non essere la constatazione dell'omaggio collettivo tributato a Fidel da milioni di cubani a Cuba, restituito con vividezza da migliaia di videoriprese, fotografie, interviste e selfie dalle strade dell'isola (e rilanciato in tutto il mondo dalle reti dei social network) nei giorni in cui la "carovana della libertà" ha condotto le ceneri del defunto dall'Avana a Santiago. Sugli organi di stampa più tenacemente "anti-castristi" ai quattro angoli del pianeta, tali immagini hanno prodotto effetti alquanto schizofrenici, nella misura in cui hanno accompagnato editoriali che continuavano, imperterriti, a raccontare di un politico solo, isolato e odiato dai suoi stessi concittadini. In generale, tuttavia, esse permettono oggi almeno di partire dal presupposto che Fidel Castro è morto circondato dal rispetto di un'ampia parte del suo popolo. D'altro canto, è opportuno confrontare le immagini provenienti da Cuba

${ }^{1}$ Per un inquadramento complessivo della rivoluzione cubana cfr. R. Gott, Cuba: A New History, Yale UP, New Haven (CT) 2005; G. Lievesley, The Cuban Revolution: Past, Present and Future Perspectives, Palgrave Macmillan, New York 2004. 
con quelle che sono giunte, negli stessi giorni, da Little Havana, il quartiere di Miami in cui si stabilì la prima generazione di fuoriusciti cubani dopo la vittoria della rivoluzione: il contrasto tra il lutto composto dell'isola e i caroselli di festa messi in scena in Florida costituisce la prova più banale della ragione per cui coloro che lasciarono Cuba all'indomani della Rivoluzione (con l'idea di riprendersela di lì a poco con la forza) siano riusciti per decenni solo a seminare morte e terrore, ma mai a fare più di mezzo passo oltre la battigia delle spiagge dell'isola. In massima parte ricchi, bianchi, oligarchici, corrotti e razzisti, i cubani della prima generazione di emigranti (e, in parte, i loro discendenti) sono stati in grado di orientare molto a lungo la politica statunitense: tuttavia, quanto alla loro pretesa di rappresentare "i cubani", essa ha avuto generalmente maggior corso presso i media occidentali che aderenza alla realtà. Sulle ragioni del relativo successo, almeno in alcune parti del mondo, di tale "dissonanza cognitiva" (in un'isola che ospita ogni anno quasi due milioni di turisti non era difficile rendersi conto del consenso, pur critico e qualificato, di cui la rivoluzione ha sempre goduto anche in anni recenti), gli storici del giornalismo potranno forse pronunciarsi in futuro. Considerata però la natura fortemente politica dell'oggetto del contendere, è difficile non avere il sospetto che, per almeno alcuni dei grandi organi di informazione internazionali, il problema non sia mai stato il possibile fallimento delle politiche di Fidel, ma il loro eventuale successo. Come diceva il personaggio interpretato da Robert Redford in Havana di Sidney Pollack (1990): l'idea di un comunismo grigio come in Unione Sovietica non era un gran problema per gli anticomunisti, ma la cosa si complicava se diventava possibile immaginarlo con la musica e i colori cubani. In questo senso, probabilmente, delegittimare Fidel Castro è stato spesso un modo per delegittimare le idee di giustizia sociale della rivoluzione cubana e l'idea stessa che un esperimento egualitario radicale potesse funzionare nel cuore e nella testa delle persone che lo vivevano.

A fare da contraltare a queste rappresentazioni, d'altro canto, vi è stata sempre una narrazione di segno opposto che, fino dai tempi della lotta contro la dittatura di Fulgencio Batista, ha attribuito a Fidel caratteristiche degne degli eroi della mitologia greca e delle figure semidivine dei culti della santería cubana. Finito sotto processo a seguito del fallito attacco alla Caserma Moncada di Santiago de Cuba del 26 luglio 1953 (la data che tradizionalmente segna l'inizio della rivoluzione), Fidel si difese con un discorso che avrebbe presentato all'opinione pubblica cubana e continentale un uomo determinato e coraggioso, che invitava il tribunale a condannarlo, chiosando: «non importa, la storia mi assolverà». Condannato a quindici anni di reclusione ma tornato in libertà nel 1955 grazie a un'amnistia proclamata anche sotto la pressione della sua popolarità, Fidel riprese la lotta armata contro la dittatura e la sua fama di abile comandante militare travalicò i confini dell'isola grazie ai reportage di una delle grandi firme del giornalismo statunitense, Herbert Matthews del «New York Times». Se poi la Sierra Maestra già offriva uno 
spettacolo verde assimilabile alla foresta di Sherwood, l'associazione tra Fidel Castro e Robin Hood divenne quasi ovvia quando Errol Flynn in persona, il volto hollywoodiano per eccellenza del leggendario bandito inglese, volle realizzare un documentario sui guerriglieri cubani.

Non pochi di coloro che ancora non si erano appassionati al personaggio furono poi convinti delle sue qualità soprannaturali alcuni giorni dopo il trionfo della rivoluzione, quando una delle colombe bianche che dovevano librarsi in volo durante il primo discorso di Fidel all'Avana preferì sistemarsi sulla sua spalla, segnalando agli astanti che gli orishas avevano indicato il loro prescelto. Infine, nel corso degli anni, i ripetuti tentativi dei servizi segreti statunitensi di assassinarlo, nonché i ripetuti fallimenti da parte dei governi di Washington di sbarazzarsi della rivoluzione cubana, hanno proiettato l'alone mitico di Fidel Castro su scala propriamente globale: si pensi alla fortuna dello slogan «Fidel seguro a los Yankees dale duro!». Anche in questo caso, tuttavia, se è necessario riconoscere che in una certa misura il "mito di Fidel" fa parte della "storia di Fidel", è anche opportuno segnalare che le rappresentazioni di Fidel Castro in chiave mitologica rischiano di consegnare alla memoria un santino immobile, fatto di virtù eterne e sempre uguali a se stesse, che ha poco a che vedere con la complessità della traiettoria storica del personaggio. Soprattutto, in modo paradossalmente simmetrico a un certo "anti-castrismo" militante, anche queste rappresentazioni rischiano di ridurre al ruolo di un'unica persona un processo storico complesso che ha coinvolto milioni di persone dentro e fuori da $\mathrm{Cuba}^{2}$.

La biografia politica di Fidel Castro è più complessa di quanto non vogliano le vulgate, che lo inchiodano da un lato al ruolo di ribelle romantico e dall'altro a quello di comunista di stretta osservanza sovietica. Nato nel 1926 in una ricca famiglia di proprietari terrieri dell'oriente cubano, fu prima un avvocato vicino alle posizioni nazionaliste del Partito ortodoxo, poi guerrigliero contro la dittatura di Batista, poi ancora capo carismatico di una rivoluzione egualitaria radicale, capo di uno stato socialista assediato dagli Stati Uniti e in rapporti di alleanza mai semplici con l'Unione Sovietica, esponente di primo piano del movimento terzomondista, infine teorico e protagonista dell'integrazione politica ed economica latinoamericana dell'inizio del XXI secolo. Attraverso queste fasi, miscele cangianti di nazionalismo, anti-imperialismo, umanesimo martiano, marxismo e marxismo-leninismo si sono composte dapprima con un sostrato di educazione borghese e gesuita, poi con le influenze della teologia della liberazione, infine con il pensiero ambientalista. In definitiva, è un percorso travagliato e non privo di contraddizioni quello che lega il giovane radicale dei primi anni '50, tanto liberale nei proclami quanto machista e caudillista nella mentalità, al periodista en jefe degli anni

${ }^{2}$ Tra le molte biografie di Fidel Castro cfr. V. Skierka, Fidel Castro: A Biography, Polity, Cambridge 2006; T. Szulc, Fidel: A Critical Portrait, Harper, New York 2000. 
successivi al ritiro dalle cariche pubbliche (avvenuto nel 2006), intento a riflettere dalle colonne del «Granma» sul carattere umanista della Rivoluzione cubana, ma anche a chiedere pubblicamente scusa (nel 2010) agli omosessuali per le pratiche gravemente discriminatorie a cui il governo rivoluzionario li aveva sottoposti negli anni '60 e '70.

Tra questi due estremi cronologici si colloca il lungo periodo in cui Fidel Castro è stato il politico comunista al centro e al timone della politica cubana, per il quale è difficile distinguere con chiarezza, almeno allo stato attuale della documentazione, ciò che delle scelte compiute all'Avana fu il prodotto genuino della volontà di Fidel stesso, e ciò che invece fu il risultato dell'adattamento a vincoli, reali o percepiti, tanto in politica interna quanto in politica estera. Se infatti la Rivoluzione si caratterizzò da subito per il suo slancio egualitario (riforma agraria, nazionalizzazione delle industrie, abolizione della discriminazione razziale, promozione del ruolo delle donne nella società, istituzione dei diritti universali alla cultura, alla salute, all'istruzione e allo sport), è opportuno mettere in luce almeno tre aspetti delle dinamiche che tra il 1959 e il 1965 resero Cuba uno stato "comunista", ciascuno dei quali contribuisce a problematizzare l'idea, assai diffusa, che la politica cubana rispondesse interamente e sempre ai disegni del suo politico più rappresentativo.

In primo luogo, l'agenda sopra accennata non apparteneva all'epoca solo a Fidel Castro ma rappresentava, al contrario, un patrimonio largamente condiviso da tutte le forze progressiste di quello che si veniva configurando come "il Terzo mondo": al livello delle élite e al livello delle masse. La campagna di alfabetizzazione del 1961, giusto per limitarsi a un esempio, fu decisa dal governo rivoluzionario ma posta in essere da migliaia di bambini, bambine, ragazzi e ragazze in età scolare a favore dei campesinos, degli operai e degli anziani dell'isola. La sua realizzazione divenne così un fatto politico e sociale destinato a restare impresso come una vera e propria epopea nella memoria collettiva cubana, ben al di là di quanto possano dire all'osservatore esterno i dati statistici, pur significativi, sulla sconfitta dell'analfabetismo.

In secondo luogo, il passo e la radicalità di alcune delle misure intraprese a Cuba (a partire dalla riforma agraria) non risultano comprensibili al di fuori del loro intreccio con le dinamiche internazionali, dalle quali anzi esse finirono spesso per essere sovradeterminate: fu, infatti, nel contesto delle relazioni interamericane dei tardi anni ' 50 che la riforma agraria cubana, meno radicale di quella che le stesse autorità statunitensi avevano promosso solo pochi anni prima nel Giappone occupato, divenne immediatamente a Washington una pietra dello scandalo e un capo d'accusa nei confronti del governo rivoluzionario dell'Avana. Sulla base di un'imponente documentazione di parte statunitense, lo storico Lars Schoultz ha mostrato come a Washington i timori reali per le idee comuniste di alcuni dei comandanti rivoluzionari si saldassero rapidamente con una consolidata cultura razzista e paternalista nei confronti dell'ex-protettorato e con gli interessi particolari di alcuni 
grandi magnati del comparto agroindustriale direttamente investiti dalla riforma, determinando già nella seconda metà del 1959 l'avvio di azioni volte a strangolare economicamente, destabilizzare politicamente e, infine, invadere militarmente l'isola ${ }^{3}$. Nel breve scorcio di tempo in cui si consumarono il boicottaggio dello zucchero cubano, l'inizio dell'embargo commerciale e l'avvio degli attentati contro le industrie e l'agricoltura dell'isola (1959-61), la fermezza della risposta dell'Avana contribuì a trasformare da locale a globale il mito della Revolución e del suo Líder maximo, ma obiettivamente si tradusse anche in scelte economico-sociali non necessariamente preventivate (in ogni caso, non secondo le forme e le tempistiche che assunsero). La riforma agraria divenne dapprima esproprio di tutte le proprietà delle grandi compagnie statunitensi, poi esproprio delle proprietà dei latifondisti cubani che, nel clima di crescente conflitto, si erano trasferiti in Florida, spesso per ingrossare le file dei gruppi di nostalgici di Batista che miravano a rovesciare il governo rivoluzionario. Una dinamica simile ebbe la nazionalizzazione delle grandi imprese, che finì per essere ben più rapida e radicale di quanto programmato, sebbene la piccola e media impresa restassero a conduzione privata fino al 1968. L'enfasi della rivoluzione su istruzione e medicina, a sua volta, è difficilmente scindibile dalla necessità pratica, che presto si presentò, di rimpiazzare i tecnici qualificati e i medici che lasciavano l'isola mano a mano che la politica di Washington faceva salire la tensione diplomatica e radicalizzava, invece di sedare, il programma economico e sociale della rivoluzione. Infine, al momento della visita all'Avana del vice-presidente del Consiglio dei ministri sovietico Anastas Mikoyan, a febbraio 1960, l'avvio dei commerci con l'Urss era certo stato messo in conto, in un'ottica terzomondista, nel nome della riduzione della dipendenza dal mercato statunitense; ma appare evidente che il successivo, rapido e radicale, inserimento di Cuba all'interno dei flussi di scambio dei paesi del Comecon (di cui l'isola divenne membro effettivo nel 1972) può essere spiegato solo sulla base del relativo successo di Washington nell'isolare l'Avana dalle relazioni politiche ed economiche emisferiche.

In terzo luogo, considerazioni simili si applicano anche alla relazione tra l'adesione di Cuba al "campo socialista" e le convinzioni di Fidel sul versante più propriamente ideologico. In parte si potrebbe qui porre il tema filosofico del potere creativo del linguaggio: già nel 1960, nella popolare canzone Cuba sì, Yanquis no, un immaginario aspirante rivoluzionario spiegava che, poiché «gli americani dic[evano] che Fidel è comunista», anche lui voleva essere considerato tale, in quanto d'accordo con Fidel. A mano a mano che saliva la tensione con l'Avana, in effetti, Fidel Castro diventò un "comunista" nel linguaggio pubblico statunitense almeno due anni prima che egli parlasse pubblicamente del «carattere socialista» della rivoluzione (aprile 1961) e

${ }^{3}$ L. Schoultz, That Little Infernal Cuban Republic, University of North Carolina Press, Chapel Hill (NC) 2009, cap. 4. 
addirittura sei anni prima che i vari movimenti e partiti che avevano guidato la rivoluzione si unissero per formare il Partito comunista cubano (1965). Più pragmaticamente, i tempi dell'adesione di Fidel Castro al marxismo (nonché la profondità della sua adesione al marxismo-leninismo "ortodosso" sovietico) sono sempre stati oggetto di discussione, anche a causa delle diverse versioni offerte dall'interessato in diverse fasi storiche: se all'inizio della sua attività politica egli proclamava fieramente di non essere un comunista e nel periodo di maggior vicinanza a Mosca proclamava, al contrario, di esserlo sempre stato, negli anni della vecchiaia ha preferito rappresentare la propria scoperta del marxismo e la propria adesione al comunismo come un processo di apprendimento progressivo, rivendicato peraltro in combinazione con una rinnovata enfasi sull'influenza del pensiero umanista del padre della patria cubana, José Martì.

È probabile che la versione più recente sia anche quella più vicina alla verità: è certo che Fidel era venuto in contatto con la letteratura marxista già all'inizio della propria militanza politica, ma tali letture non erano state sufficienti a farlo aderire al Partido socialista popular, il partito cubano più vicino alle posizioni comuniste. In compenso, anche dopo lo sbarco del Granma e l'avvio della guerriglia dei barbudos sulla Sierra maestra (dicembre 1956), la dirigenza di questo partito mantenne a lungo una posizione apertamente scettica nei confronti di quelli che, seguendo le indicazioni di Mosca, considerava essenzialmente degli avventurieri piccolo-borghesi (solo sul finire del 1958, quando la vittoria della rivoluzione era ormai una prospettiva concreta, tra il Movimiento 26 Julio di Fidel e i comunisti si stabilì un rapporto di collaborazione, peraltro non privo di tensioni). Come già accennato, tuttavia, dal trionfo della rivoluzione avrebbero poi dovuto passare quasi altri due anni e mezzo prima che Fidel affermasse il «carattere socialista» della rivoluzione stessa, il 16 aprile 1961: in merito a tale svolta linguistica, sebbene si possa affermare che molto di ciò che la rivoluzione stava facendo rispecchiava quanto scritto nei programmi classici del socialismo novecentesco (tanto che essa attrasse la simpatia dei socialdemocratici europei prima ancora di quella di Mosca), è impossibile non ricordare che, mentre Fidel parlava, la Cia stava dando inizio al (disastroso) tentativo di invasione della Baia dei porci (17-19 aprile 1961).

Su cosa poi il socialismo sia e debba essere sul piano strettamente economico, all'analisi storica dovrebbero risultare piuttosto chiare almeno due conclusioni: da un lato, la fiducia nella pianificazione di tipo sovietico che Fidel Castro abbracciò negli anni ' 70 e ' 80 trova una continuità solo parziale nelle riflessioni più recenti, in cui l'elemento pubblico, quello privato e quello cooperativo convivono in misura assai più bilanciata, mentre l'enfasi sulla produzione è temperata dalle preoccupazioni per il cambiamento climatico, con toni che uniscono antiche parole d'ordine terzomondiste sulla necessità di un nuovo ordine economico internazionale a echi di dibattiti europei più recenti 
sulla «decrescita felice». Dall'altro, anche se proposte (soprattutto negli anni '70) come modelli validi erga omnes, tali teorizzazioni sono state, almeno in parte, anche tentativi di razionalizzare ex post quello che all'economia cubana (piccola, ex-coloniale e sotto embargo) è stato di volta in volta imposto dall'economia internazionale. Per limitarsi a un esempio, la prima reazione del governo cubano alle difficoltà economiche degli anni '80 fu la "rettificazione degli errori" della pianificazione, mentre l'introduzione di elementi di "economia mista" iniziò, obtorto collo, solo dopo che il crollo del blocco socialista aveva privato Cuba dei propri partner commerciali e delle sovvenzioni sovietiche ${ }^{4}$.

La distinzione, almeno concettuale, tra ciò che nella storia cubana è avvenuto per volontà di Fidel Castro, ciò che è avvenuto anche per volontà di Fidel Castro e ciò che è avvenuto nonostante la volontà di Fidel Castro dovrebbe impegnare gli storici futuri in un serio programma di ricerca relativo ai punti più controversi della sua azione politica. Allo stato delle ricerche, è probabilmente la politica estera cubana ad aver ricevuto l'attenzione storiografica più approfondita. In particolare, il contrastato rapporto con gli Stati Uniti pare essere stato tale essenzialmente per una mancanza di interlocutori a Washington (con la parziale eccezione di Jimmy Carter negli anni '70 e, in misura assai più netta, di Barack Obama dopo il 2009). D'altro canto, è legittimo chiedersi anche in quale misura Fidel abbia desiderato che i rapporti con Mosca fossero tanto stretti quanto quelli che Cuba intrattenne tra gli anni '60 e la fine degli anni '80. Sarebbe superficiale affermare che l'adesione al blocco socialista fosse per Cuba una misura strumentale per difendersi dall'assedio statunitense: al contrario, come è stato ampiamente documentato, il modello sovietico suscitò in tutto il Terzo mondo un'ampia fascinazione per la modernizzazione rapida che esso prometteva. La dirigenza cubana non faceva eccezione, a prescindere dalle relazioni con gli Usa ${ }^{5}$.

Allo stesso tempo, sono numerosi gli episodi che indicano nel rapporto tra Cuba e l'Urss una dinamica tutt'altro che semplice, dalle proteste con cui fu accolto nel 1962 il raggiungimento dell'accordo tra Chruščëv e Kennedy (senza consultazione con la parte cubana) per porre termine alla cosiddetta "crisi dei missili di ottobre", all'attivismo "antimperialista" nel Terzo Mondo nel corso degli anni '70, quando Mosca era invece impegnata nella promozione della "distensione" con Washington. In merito, e contro una vulgata dura a morire, le ricerche di Piero Gleijeses hanno già mostrato, sulla base dei documenti d'archivio cubani, la sostanziale autonomia da Mosca con cui il governo dell'Avana agì in Africa australe, sconfiggendo ripetutamente sul

${ }^{4}$ Cfr. le riflessioni in F. Castro-I. Ramonet, Cien Horas con Fidel, Consejo de Estado, La Habana 2006.

${ }^{5}$ O.A. Westad, The Global Cold War. Third World Interventions and the Making of Our Times, Cambridge UP, Cambridge 2005. 
campo di battaglia l'esercito sudafricano (1976 e 1987) e aprendo la strada al consolidamento dell'indipendenza angolana, alla liberazione della Namibia e alla caduta dello stesso regime dell'apartheid a Pretoria. D'altro canto, i limiti percepiti di tale margine di autonomia non furono oltrepassati quando, pur tra molti dubbi, Fidel Castro offrì a Mosca la sua ben meno edificante approvazione alla repressione della Primavera di Praga ${ }^{6}$.

Analogamente, per la politica interna la storiografia ha già saputo mostrare che la storia politica e sociale della Cuba rivoluzionaria ha attraversato fasi diverse, smentendo così l'idea, ancora diffusa nel dibattito politico, di una realtà immobile e sempre uguale a se stessa. Sarà tuttavia necessario comprendere in quale misura l'innegabile propensione di Fidel all'accentramento delle responsabilità, il richiamo ideologico al modello sovietico e la condizione obiettiva di "cittadella assediata" abbiano contribuito a rendere Cuba un sistema a partito unico dopo il 1965 (pur con le istanze di democrazia partecipativa e le elezioni su base apartitica per l'Assemblea nazionale del potere popolare, introdotte dalla Costituzione del 1976).

$\mathrm{Su}$ un terreno vicino, considerata l'enfasi che la rivoluzione ha sempre messo sulla produzione artistica e culturale e sulla "battaglia delle idee", domande simili dovrebbero porsi anche rispetto alla marginalizzazione, l'incarceramento e spesso, successivamente, l'esilio di centinaia di oppositori politici (reali o presunti) nel corso degli anni '70 - un periodo che gli stessi manuali di storia cubani chiamano oggi il «quinquennio grigio»-e, pur se in misura assai minore, nei decenni successivi. D'altro canto, la storia della $\mathrm{Cu}-$ ba rivoluzionaria offre forse oggi, in pieno sviluppo degli studi post-coloniali e della storia globale, anche la possibilità di decolonizzare gli immaginari, decentrandoli in una qualche misura rispetto alle tipiche priorità dei maschi adulti bianchi di classe agiata. All'immagine stantia di una Cuba in cui «non c'è libertà ma la sanità e l'istruzione sono gratuite» è forse il caso di sostituire quella di un'isola in cui l'istruzione è certo di primo livello, la sanità mantiene con grande difficoltà un livello qualitativo dignitoso, la dieta è piuttosto povera e la libertà di stampa, di associazione e di critica hanno limiti obiettivi, ma in cui la popolazione può godere di molte libertà che altrove sono tutt'altro che scontate, tra cui quella di non lavorare tutta la vita per vivere (e magari quella di non dipendere da un voucher per sopravvivere), quella di decidere sulle proprie pratiche sessuali e riproduttive, quella di non essere aggrediti dalla polizia a causa del colore della propria pelle, quella di attraversare un parco urbano di notte senza alcun rischio per la propria incolumità,

${ }^{6}$ P. Gleijeses, Conflicting Missions. Havana, Washington and Africa (1959-1976), University of North Carolina Press, Chapel Hill (NC) 2002; Id., Visions of Freedom. Havana, Washington, Pretoria and the Struggle for Western Africa (1976-1991), University of North Carolina Press, Chapel Hill (NC) 2013. Nel 1991 Cuba fu il primo paese straniero visitato da Nelson Mandela dopo il rilascio dalla lunga detenzione. 
quella di pregare il dio che si preferisce (o gli dei che si preferiscono, o di non pregare affatto) $o$, più banalmente, quella di poter fare festa in compagnia. Una lettura comparata dei country profile dei paesi delle Americhe (del Nord e del Sud) stilati da Amnesty International negli ultimi anni è, in questo senso, un esercizio istruttivo.

Un giorno la figura di Fidel Castro assumerà caratteri più storici e meno mitici (o meno demoniaci, a seconda dei punti di vista) di quelli tramandati finora dal dibattito sul suo conto, non sovrapponibili interamente né con il giovane politico che intimò agli artisti «dentro la rivoluzione tutto, fuori dalla rivoluzione niente», né con l'anziano politico che inaugurò un monumento a John Lennon all'Avana, affermando di essere un sognatore proprio come il musicista dei Beatles. Già da ora è possibile però affermare che Fidel Castro è stato soprattutto un uomo della sinistra novecentesca, con lo sguardo coerentemente puntato sull'uguaglianza sociale come fonte di ogni emancipazione, la parola incentrata sulla fratellanza come tratto di originalità del modello cubano e la mente occupata da istanze di libertà in cui i soggetti collettivi ("i lavoratori", "gli umili", "le donne", "i popoli", "i cubani e le cubane") avevano la precedenza su quelli individuali. Le manifestazioni di lutto a Cuba e i caroselli in Florida che hanno accompagnato la sua morte hanno forse scritto la prima pagina del giudizio storico su di lui. Il contenuto delle pagine che restano da scrivere dipenderà in parte da quanto gli storici riusciranno a chiarire degli episodi più controversi della sua biografia politica, in parte da come i suoi eredi a Cuba sapranno gestire le prossime sfide istituzionali e politiche dell'isola e, in parte, da come un sistema politico-economico mondiale in cui otto individui possiedono un patrimonio equivalente a quello dei tre miliardi e mezzo di abitanti più poveri del pianeta saprà affrontare le sfide sociali ed ecologiche del futuro. 